\title{
Concise guidance: diagnosis and management of polymyalgia rheumatica
}

\author{
Bhaskar Dasgupta on behalf of the Polymyalgia Rheumatica Guideline Development Group*
}

\begin{abstract}
Polymyalgia rheumatica (PMR) is among the most common reasons for long-term steroid prescription with great heterogeneity in presentation, response to steroids and disease course. The British Society for Rheumatology and the British Health Professionals in Rheumatology have recently published guidelines on management of PMR. The purpose of this concise guidance is to draw attention to the full guidelines and provide a safe and specific diagnostic process with advice on management and monitoring - specifically targeted at general practitioners, general physicians and rheumatologists.
\end{abstract}

KEY WORDS: diagnosis, polymyalgia rheumatica, steroid therapy, treatment

\section{Introduction and aims}

Polymyalgia rheumatica (PMR) is one of the most common inflammatory rheumatic diseases of the elderly and represents one of the frequent indications for long-term corticosteroid therapy in the community. Its management is currently subject to wide variations of clinical practice and it may be managed in primary or secondary care by general practitioners (GPs), rheumatologists and non-rheumatologists.

The diagnosis of PMR can be challenging, as many clinical and laboratory features may also be present in other conditions, including other rheumatological diseases, infection and neoplasm. Also, the response to standardised therapy is heterogeneous, and a significant proportion of patients do not respond completely.

\section{Aims of the guideline}

The objective of this concise guidance is to produce a summary of the longer British Society for Rheumatology and British Health Professionals in Rheumatology guidelines ${ }^{1}$ and to provide recommendations in the following areas:

Bhaskar Dasgupta, consultant in rheumatology, honorary professor, Essex University Southend University Hospital, Westcliff-on-Sea, Essex

Concise Guidance to Good Practice Series editors: Lynne Turner-Stokes and Bernard Higgens

*For membership of the guideline development group please see the full guideline ${ }^{1}$
- $\quad$ safe and more accurate diagnosis of patients presenting with polymyalgic symptoms

- appropriate referral for specialist assessment and management

- appropriate corticosteroid dosage regimens

- bone protection to reduce the common morbidity of osteoporotic fractures

- appropriate follow-up and monitoring procedures.

Information in this concise guidance has been extracted from the full guideline. ${ }^{1}$ Please refer to the full guidance for details of the methodology. The guidelines were developed using the AGREE criteria and Scottish Intercollegiate Guidelines Network guidance was used to grade the recommendations.

\section{Challenges in the diagnosis of polymyalgia rheumatica}

The cardinal features of PMR - proximal pain and stiffness with a raised acute phase response - are familiar to most physicians. However, the diagnostic presentation is varied and this may lead to diagnostic error:

- proximal pain and stiffness can occur in many other illnesses

- a third of patients have systemic symptoms such as fever, anorexia and weight loss ${ }^{2}$

- a considerable number (15-30\%) may have distal musculoskeletal manifestations such as peripheral arthritis, distal swelling with pitting oedema and carpal tunnel syndrome.

PMR is also associated with giant cell arteritis (GCA) in 10\% of the cases and up to $50 \%$ of cases of GCA may have polymyalgic symptoms at presentation. ${ }^{2}$ Moreover, an acute phase response can occur in other settings such as other rheumatological conditions, neoplasia and infection.

Many clinicians and two empirically developed sets of diagnostic criteria use a response to corticosteroids as the main defining feature of this condition. ${ }^{3}$ This, too, may encourage diagnostic error, since corticosteroids are potent anti-inflammatory agents that can mask symptoms from a host of serious conditions ranging from osteoarthritis, rotator cuff problems, rheumatoid arthritis, cancer and infection, especially if used in high doses and for protracted lengths of time.

Studies have shown a revision of the initial diagnosis on follow up in several cases. The list includes infection, neoplasia, inflammatory arthritis and connective tissue diseases. ${ }^{4}$ 


\section{The recommendations}

\section{Recommendations}

\section{Grade}

1 Diagnostic approach

A safe stepped diagnostic process should be adopted for the evaluation of polymyalgia rheumatica (PMR) (see Fig 1).

This consists of:

1 assessment for core inclusion and core exclusion features (see Steps 1 and 2)

2 exclusion of mimicking conditions (see Fig 2)

3 assessment of the response to a standardised dose of $15 \mathrm{mg}$ prednisolone

4 confirmation of diagnosis at early follow up.

Note: Active giant cell arteritis (GCA) is excluded from this guideline, as immediate institution of high-dose steroid therapy is necessary.

2 Investigations

The appropriate investigations should be performed prior to corticosteroid commencement:

- a core dataset of required investigations is given in Fig 1.

\section{Specialist referral}

The following patients should be referred for specialist evaluation.

Atypical clinical presentations:

- younger patient $<60$ years

- chronic onset

- lack of shoulder involvement

- lack of inflammatory stiffness

- 'red flag' features: prominent systemic features, weight loss, night pain, neurological signs

- peripheral arthritis or other features of autoimmune or muscle disease

- normal or very high inflammatory markers (ie erythrocyte sedimentation rate/C-reactive protein (ESR/CRP) $>100)$

Treatment dilemmas:

- incomplete or non-response to corticosteroids

- ill-sustained response

- unable to reduce corticosteroid dose

- need for prolonged corticosteroid therapy ( $>2$ years)

- contra-indications to corticosteroid therapy.

4 Corticosteroid therapy

Patients should be given a course of oral or intramuscular steroid treatment. Suggested regimens are given below.

Note: In the absence of GCA, urgent steroid therapy is not indicated before clinical evaluation is complete.

Suggested initial oral steroid and tapering regimen:

- prednisolone $15 \mathrm{mg}$ daily for three weeks

- then $12.5 \mathrm{mg}$ daily for three weeks

- then $10 \mathrm{mg}$ daily for four to six weeks

- then reduction by $1 \mathrm{mg}$ every four to eight weeks OR alternate day reductions (eg $7.5 \mathrm{mg} / 10 \mathrm{mg}$ alternate days).

Intramuscular methylprednisolone may be used in milder cases and may reduce the risk of steroid-related complications:

- initial dose is $120 \mathrm{mg}$ every three to four weeks

- reducing by $20 \mathrm{mg}$ every two to three months. ${ }^{5}$

However, the approach to treatment must be flexible and tailored to the individual, with regards to disease activity, steroid toxicity and patient wishes.

Duration of steroid therapy:

- this is usually one to three years

- some may require small doses beyond this

- steroids may be stopped if continuing lack of inflammatory symptoms

- raised ESR/CRP without clinical symptoms is not an indication to continue corticosteroids. 


\section{The recommendations - continued}

5 Other therapies

Bone and stomach protection should be co-prescribed with corticosteroids (see Royal College of Physicians guidance on steroid-induced osteoporosis 6 ).

\section{Monitoring and follow up}

Early follow up is necessary as part of the diagnostic process, and should occur at one to three weeks post steroid commencement.

Follow-up schedule

The suggested follow-up schedule is:

- weeks $0,1-3,6$

- months 3, 6, 9, 12 in first year (with extra visits for relapses or adverse events).

Clinical and laboratory monitoring

The following should be assessed at each monitoring visit.

Clinical:

- disease activity and response to treatment

- complications of disease, including GCA and large vessel vasculitis

- steroid-related side effects

- atypical features or alternative pathology

- persistent pain may arise from degenerative or soft tissue pathology (eg rotator cuff tears).

Laboratory:

- full blood count

- ESR/plasma viscosity or CRP

- urea and electrolytes

- glucose or urine dipstick.

7 Relapse

Relapse is the recurrence of PMR symptoms or the onset of GCA, not just unexplained raised ESR/CRP.

Treatment of relapse:

- clinical features of GCA

- treat as GCA (usually 40-60 mg prednisolone daily).

- clinical features of PMR

- increase prednisolone to previous higher dose

- single dose of intramuscular methylprednisolone $120 \mathrm{mg}$ can also be used.

- further relapses

- consider adjuvant immunosuppressive therapy after two relapses.

Note: This treatment should only be given under specialist care.

\section{Definitions and diagnosis}

Diagnosis of PMR should start with evaluation for core inclusion and exclusion criteria, the exclusion of mimicking conditions, and a core dataset of laboratory investigations to be performed before treatment. These are outlined in Fig 1. A decision-making approach to proximal pain and stiffness is given in Fig 2.

This is followed by the assessment of the response to a standardised daily dose of $15 \mathrm{mg}$ prednisolone. In PMR, a clinical response of $>70 \%$ is expected at one week, with normalisation of laboratory inflammatory markers at four weeks.
An atypical clinical presentation or failure to respond to steroid should prompt the consideration of alternative pathology, and specialist referral. Active GCA is excluded from this guideline, as immediate institution of high-dose steroid therapy is necessary. ${ }^{1}$ GCA will be the subject of a future set of concise guidance.

\section{Implications for implementation}

There are no cost implications for implementation of these guidelines apart from relevant training for GPs who should also have access to the routine investigations outlined and the ability to follow up as suggested. 


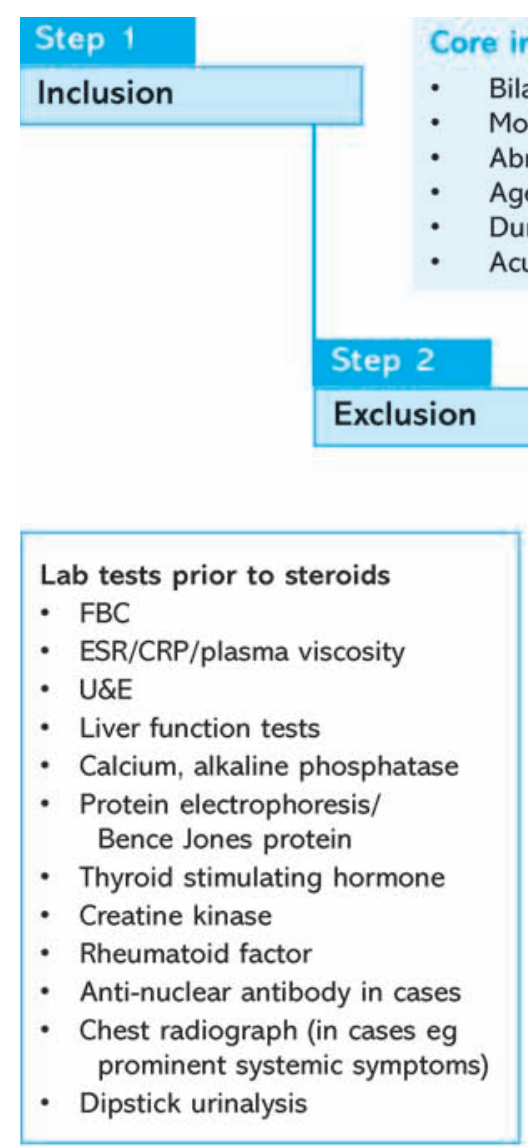

Early specialist referral is recommended for:

- patients with atypical features or features that increase likelihood of a non-PMR diagnosis:

- younger patients $<60$ years

- chronic onset

- lack of shoulder involvement

- lack of inflammatory stiffness

- 'red flag' features:

prominent systemic features, weight loss, night pain, neurological signs

- peripheral arthritis or other features of CTD/ muscle disease

- normal or very high ESR/CRP

- treatment dilemmas such as:

- incomplete or non-response to corticosteroids

- ill-sustained response to corticosteroids

- unable to reduce corticosteroids

- contra-indications to corticosteroid therapy

\section{Core exclusion criteria}

- Active cancer

- Infection

- GCA

Other inflammatory conditions:

- RA, other arthropathies

- SLE, myopathies, other connective tissue diseases

Non-inflammatory:

- Local shoulder and hip conditions

- Fibromyalgia/pain syndromes

- Osteoarthritis

Endocrine eg thyroid

Drug induced eg statins

Statin-related myalgia/myopathy

\section{Step 3}

\section{Low-dose steroids}

\section{Step 4}

Prednisolone 15-20 mg daily

Clinical response in 1 week

- At least $70 \%$ global improvement

- Lab resolution of acute phase response in 3-4 weeks

Relapses

- Increase steroids to previous higher dosage first and second relapse

- Consider immunosuppressive eg methotrexate

GCA relapse

- Treat with high dose steroids 40-60 mg prednisolone

- Consider alternative diagnosis with steroid partial or non-response

Symptoms to monitor

- Proximal pain

- Morning stiffness

- Disability related to the PMR

- Adverse events

- Osteoporotic risk

- Symptoms that may suggest an alternative diagnosis

\section{PMR}

- Gradual steroid tapering

IM depomedrone in mild cases, or

contraindications to oral steroids

Bone protection

- U\&E

- Glucose (every 3 months)

- FBC

Fig 1. A referral pathway for polymyalgia rheumatica (PMR) compatible with these guidelines is suggested in the Map of Medicine. CRP = C-reactive protein; CTD = connective tissue disease; ESR = erythrocyte sedimentation rate; FBC = full blood count; $\mathrm{GCA}=$ giant cell arteritis; IM = intramuscular; RA = rheumatoid arthritis; SLE = systemic lupus erythematosus; U\&E = urea and electrolytes. 
Fig 2. Recommendation on approach for the evaluation of proximal pain and stiffness. PTH = parathyroid hormone; RA = rheumatoid arthritis; RS3PE = remitting, seronegative symmetrical synovitis with pitting edema; SLE $=$ systemic lupus erythematosus; TSH = thyroid stimulating hormone.

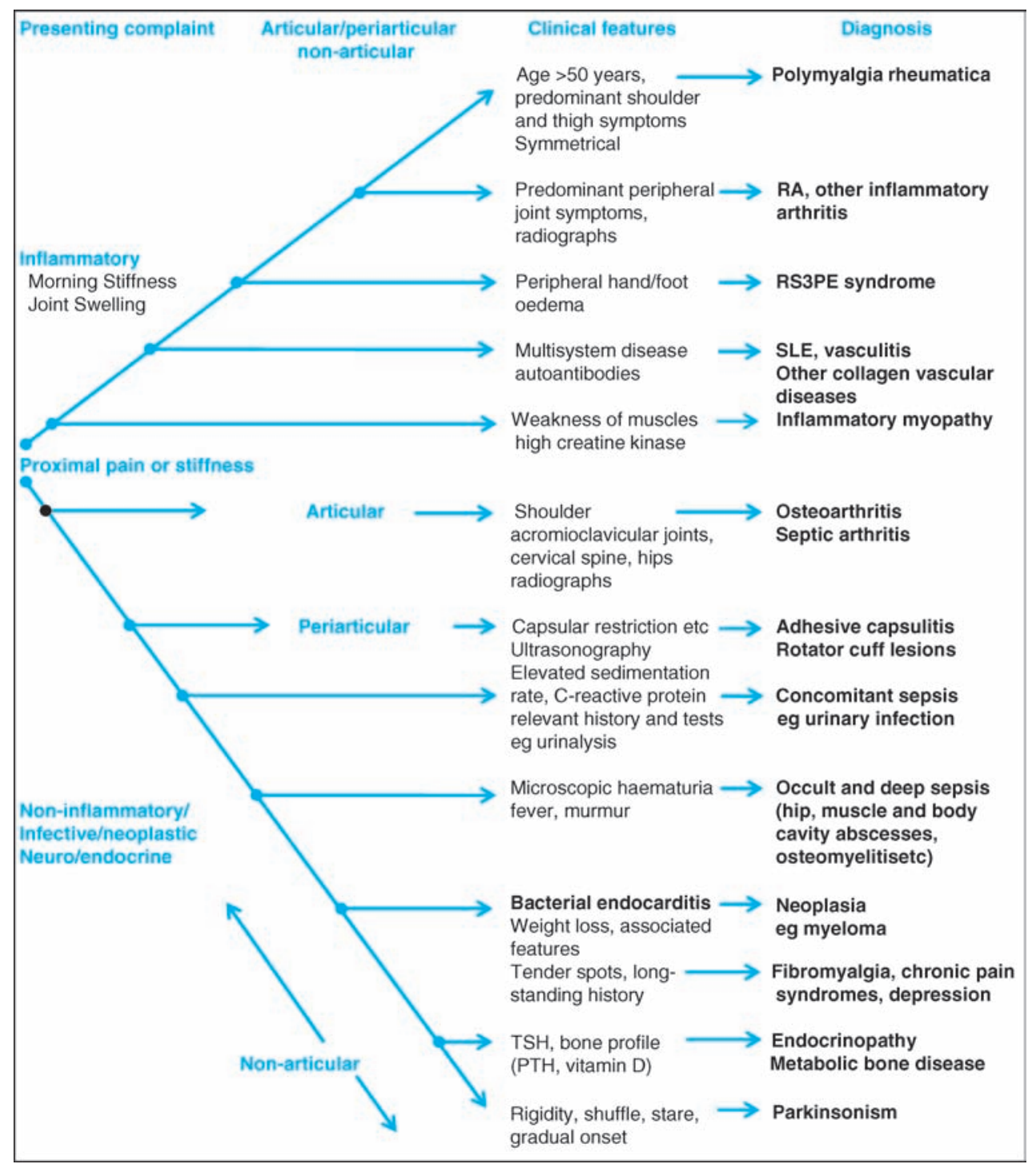

\section{Further information}

Further information on the guidelines, ie references, guideline development process, implications and implementation, is available at Rheumatology online (www.rheumatology.oxford journals.org). ${ }^{1}$

\section{References}

1 Dasgupta B, Borg FA, Hassan N et al. BSR and BHPR guidelines for the management of polymyalgia rheumatica. Rheumatology 2010;49:186-90.

2 Dasgupta B, Kalke S. Polymyalgia rheumatica. In: Isenberg D, Maddison P, Woo P, Glass D, Breedveld F (eds), Oxford textbook of rheumatology, 3rd edn. Oxford: Oxford University Press, 2004:977-82.
3 Dasgupta B, Hutchings A, Matteson EL. Polymyalgia rheumatica: the mess we are in and what we need to do about it. Arthritis Rheum 2006;55:518-20.

4 Hutchings A, Hollywood J, Lamping D et al. Clinical outcomes, quality of life and diagnostic uncertainty in the first year in polymyalgia rheumatica. Arthritis Rheum 2007;57:803-9.

5 Dasgupta B, Dolan AL, Fernandes L, Panayi G. An initially doubleblind controlled 96 week trial of depot methylprednisolone against oral prednisolone in the treatment of polymyalgia rheumatica. Brit J Rheumatol 1998;37:189-95.

6 Royal College of Physicians. Glucocorticoid-induced osteoporosis: guidelines for prevention and treatment. London: RCP, 2002.

Address for correspondence: Professor B Dasgupta, Department of Rheumatology, Southend University Hospital, Prittlewell Chase, Westcliff-on-Sea, Essex SSO ORY. Email: bhaskar.dasgupta@southend.nhs.uk 